\title{
DYNAMICAL ANALYSIS OF STIFFENED PLATES UNDER PATCH LOADING
}

\author{
A.K.L. SRIVASTAVA* \\ Civil Engineering Department \\ N. I. T. Jamshedpur- 831014, INDIA \\ E-mail: aklsriv.nitjsr@yahoo.com \\ S.R. PANDEY and A. KUMAR \\ Research Scholar \\ N.I.T. jamshedpur-831014, INDIA
}

\begin{abstract}
The vibration characteristics of stiffened plates with cutouts subjected to in-plane partial edge loadings at one end at the plate boundaries are studied using the finite element method. Buckling loads and vibration frequencies are determined for different cutout ratios and extent of partial edge loading at one end. In the structural modelling, the plate and the stiffeners are treated as separate elements where the compatibility between these two types of elements is maintained. The main elegance of the formulation lies in the treatment of the stiffeners. The stiffeners can be placed anywhere within the plate element, and need not be placed on the nodal lines. The vibration characteristics are discussed and the results are compared with those available in the literature. Numerical results are presented for a range of cutout to plate size from 0 to 0.8 .
\end{abstract}

Key words: FEM, partial edge loadings, buckling load, vibration frequency, stiffener.

\section{Introduction}

Structures consisting of thin plates are often reinforced with stiffening ribs for achieving greater strength with relatively less material and thus making the structure quite efficient. The wing and the fuselage of an aircraft consist of a thin skin with an array of stiffeners. The hull of a ship, its deck and superstructure, a road bridge and a launching pedestal of rocket are examples of stiffened plate structures. With such a wide range of applications, the stiffened plates are often subjected to dynamic in-plane loads of varying magnitude and complexity.

Plates with cutout are commonly encountered in many technological situations, aeronautical, civil, mechanical and naval engineering. Plates with interior openings are often used in industrial design such as machines, bridges, aircraft etc. Cutouts are used in most of the situation due to operation conditions, passage of ducts and conduits, cables etc. However when interior holes are cut from a plate structures, the mechanical behaviour of the plate structures is changed. To avoid induced acoustic noise and/or mechanical failure due to mechanical resonance, vibration problems of such plate structure can be studied.

Vibration problems of plates with internal cutouts or supports are commonly encountered in the engineering practice. In aeronautical, marine and civil structural design, cutouts are deliberately incorporated to reduce material, lighten the loads, and provide ventilation and to alter the resonant frequencies of the structures.

The buckling and vibration instability of stiffened plates with cutouts subjected to uniform and nonuniform in-plane edge loadings are of considerable importance. Aircraft wing skin panels, which are made of thin sheets, are usually subjected to non-uniform in-plane stresses causing partial edge loading at the edges. These elements, being thin are highly susceptible to the buckling instabilities under such loading. In contrast to high transverse strength, they often lose stability at fairly low stress levels, when subjected to in-plane forces.

\footnotetext{
* To whom correspondence should be addressed
} 
Cutouts in aerospace, civil, mechanical and marine structures are inevitable mainly for practical and design considerations. Free edges of the cutout are susceptible to instability behaviour due to local buckling. The buckling and vibration characteristics of structures with cutouts pose a tremendous challenge. The instability effects are improved with the provision of stiffeners. The study of static stability of stiffened plate structures with or without cutout is of interest for the present work.

A large number of references in the published literature deal with the buckling, vibration behaviour of rectangular plates subjected to in-plane uniform loading. The problem becomes complicated when the loading is partially loaded over the edges. Relatively fewer investigations deal with the static stability problems under in-plane partial edge loadings.

Yamaki (1953) analyzed the plate subjected to locally distributed in-plane loading over a finite length of the edge at the center of two opposite edges. Leggett (1937) was able to obtain an approximate non-uniform in-plane stress distribution within the plate subjected to point loading at the two opposite edges. The vibration of flat thin plates subjected to complex middle force system under arbitrary stress system based on Kirchoff's theory is studied by Mei and Yang (1972). Vibration and buckling calculation for rectangular plates subjected to complicated in-plane stress distribution by using numerical integration in a Raleigh-Ritz analysis is studied by Dickinson and Kalidas (1981).

Sundersan et al (1998) studied the influence of partial edge compression on buckling behaviour of angle ply plates for few orientations. Vibration and buckling calculations for rectangular plates subjected to non-uniform in-plane stress distribution were studied by Deolasi and Datta (1995).

Vibration and dynamic stability of stiffened plates subjected to in-plane uniform harmonic edge loading is studied using the finite element analysis by Srivastav et al. (2002).

A number of investigations exist on buckling under uni-axial uniform loads of stiffened plates having longitudinal equispaced stiffeners, mostly based on shear deformation theories under different classical boundary conditions.

A finite element analysis of a clamped thin plate with different cutout sizes, along with experiments was carried out by Monahan et al. (1970) using holographic interferometry. Ritchie and Rhodes (1975) investigated theoretically and experimentally the behaviour of simply supported uniformly compressed rectangular plates with central holes, using a combination of Rayleigh-Ritz and finite element methods. Paramsivam and Sridhar Rao (1969) developed a finite difference method for obtaining the natural frequencies and mode shapes for rectangular plates, of varying stiffnesses causing re-entrant corners, by assuming average curvature at the corners.

Ali and Atwal (1980) studied the natural frequencies of simply supported rectangular plates with rectangular cutouts using the Rayleigh Ritz method. Using the finite element method for computing in-plane stresses, Uenoya and Rewood (1986) determined the shear buckling of square plates with holes by the applying Rayleigh- Ritz method.

Mundkur et al. (1994) studied the vibration of square plates with square cutouts by using boundary characteristics orthogonal polynomials satisfying the boundary conditions.

Paramsivam and Sridhar Rao (1973) modified the grid framework model suitably to obtain the natural frequencies of a square plate with stiffened square openings. The free vibration characteristics of unstiffened and longitudinally stiffened square panels with a square cutout are investigated by Sivasubramonian et al. (1977) using the finite element method. Lam et al. (1990) studied the vibration of a rectangular plate by dividing the total domain into smaller areas and using a modified form of the RayleighRitz method. The same method was employed by Lam and Hung to study the vibrations of plates with stiffened openings using orthogonal polynomials and partitioning method

However, little attention has been paid to buckling, vibration behaviour of stiffened plates with / without cutout subjected to partial edge loading at the plate boundary of practical interest. The vibration and stability analysis of stiffened plates with / without cutout subjected to partial loading is sparsely treated and can be extended to practically important patch loading under different boundary conditions. The authors could not find any work in the literature on the buckling and vibration of a stiffened plate with cutout subjected to in-plane partial edge loading at the plate boundary. Thus the study on buckling and vibration of a stiffened plate subjected to partial edge loading is new. 
The present paper deals with buckling and vibration analysis of eccentrically stiffened plates with cutout subjected to harmonic in-plane partial edge load. A finite element formulation is applied to study the effects of different boundary conditions, cutout size, extent of partial edge loading position.

In the present analysis, the plate is modelled with the nine noded isoparametric quadratic elements with five degrees of freedom, where the contributions of bending and membrane actions are taken into account. One of the advantages of the element is that it includes the effect of shear deformation and rotary inertia in its formulation. Thus the analysis can be carried out for both thin and thick plates. The formulation of the stiffener is mode in such a manner so that it may lie anywhere within a plate element.

\section{Governing equations and proposed finite element}

The governing equations for free vibrations are

$$
[M]\{\ddot{q}\}+\left[K_{b}\right]\{q\}=\{0\} .
$$

The governing equations for vibrations with in-plane loads are

$$
[M]\{\ddot{q}\}+\left[\left[K_{b}\right]-P\left[K_{G}\right]\right]\{q\}=\{0\} .
$$

The matrix $\left[K_{G}\right]$ is a function of external in-plane loads.

The governing equations for vibration with in-plane loads are

$$
\left[\left[K_{b}\right]-P\left[K_{G}\right]\right]\{q\}=\{0\}
$$

where $\left[K_{b}\right],\left[K_{G}\right],[M]$ are the overall elastic stiffness, geometric stiffness, and mass matrices, respectively, $\{q\}$ is the displacement vector. The elements of overall matrices in Eqs (2.1), (2.2) and (2.3) can be generated through the assembly of corresponding element matrices. The derivation of each of the above matrices is given in the following section. The eigenvalues of the above equations give the natural frequencies and buckling loads for different modes. The lowest values of frequency and buckling loads are termed as the fundamental frequency and fundamental critical load of the structure.

The overall elastic stiffness matrix, geometric stiffness matrix and mass matrix are generated from the assembly of those element matrices and stored in a single array where the variable bandwidth profile storage scheme is used. The eigenvalues is solved by the simultaneous iteration technique proposed by Corr and Jennings (1976).

For problems involving complex in plane loading and boundary conditions, analytical methods are not easily adaptable and numerical methods like the finite element method (FEM) are preferred. The formulation is based on Mindlin's plate theory, which will allow for the incorporation of shear deformation. The plate skin and the stiffeners are modelled as separate elements but the compatibility between them is maintained. The middle plane of the plate is taken as the reference plane for both the plate and the stiffeners. The nine noded isoparametric quadratic elements with five degrees of freedom $\left(u, v, w, \theta_{X}\right.$ and $\left.\theta_{y}\right)$ per node have been employed in the present analysis.

As the plate element for a stiffened plate includes in-plane displacements, the formulation presented here includes both in- plane and bending displacements. The in-plane displacements $u$ and $v$ need to be considered only when the stiffeners are connected eccentrically to the plate. The effect of in-plane deformations is taken into account in addition to the deformations due to bending, which will help to model the stiffener eccentricity conveniently. The element matrices of the stiffened plate element consist of the contribution of the plate and that of the stiffener. A study of the stiffness and mass matrices of the stiffener element reveals that the contribution of the beam element is reflected in all 9 nodes of the plate element, which contains the stiffener, and they are coupled also. This is achieved through the expression of the strain displacement relationship in terms of displacement of 9 nodes of the plate. The contribution of the stiffener 
to a particular node depends on the proximity of the stiffener to that node. This is similar to the concept proposed by Mukherjee and Mukhopadhyay (1990). The whole domain of interest (plate area) is divided into a number of finite elements. The formulation is based on the following assumptions.

\section{Assumptions of the analysis}

1. The material of the plate and the stiffener obeys Hook's law.

2. The bending deformation follows Mindlin's hypothesis; therefore the linear elements perpendicular to the middle plane of the plate before bending remain straight, but not necessarily normal to the middle plane of the plate after bending.

3. The deflection of the points of the middle plane of the plate in the direction normal to this plane is small in comparison to the thickness of the plate.

4. The normal common to the plate and the stiffeners before bending remain straight after bending.

5. The deflection in the $z$ direction is a function of $x$ and $y$ only.

6. The transverse normal stresses are neglected.

\subsection{Formulation of plate element for eccentrically stiffened plate}

The strain displacement relation can be written by

$$
\{\varepsilon\}=\sum\left[B_{p}\right]_{r}\{\delta\}_{r}=\left[B_{p}\right]\{\delta\} .
$$

Element stiffness matrix is expressed as

$$
\left[K_{p}\right]=\int_{-1}^{+1} \int_{-1}^{+1}\left[B_{p}\right]^{T}\left[D_{p}\right]\left[B_{p}\right]\left|J_{p}\right| d \xi d \eta
$$

where

$$
\begin{aligned}
& {\left[B_{P}\right]=\left[\begin{array}{llllll}
{\left[B_{P}\right]_{1}} & {\left[B_{P}\right]_{2}} & \cdots & {\left[B_{P}\right]_{r}} & \cdots & {\left[B_{P}\right]_{9}}
\end{array}\right],} \\
& {\left[B_{P}\right]_{r}=\left[\begin{array}{ccccc}
\frac{\partial N_{r}}{\partial x} & 0 & 0 & 0 & 0 \\
0 & \frac{\partial N_{r}}{\partial y} & 0 & 0 & 0 \\
\frac{\partial N_{r}}{\partial y} & \frac{\partial N_{r}}{\partial x} & 0 & 0 & 0 \\
0 & 0 & 0 & -\frac{\partial N_{r}}{\partial x} & 0 \\
0 & 0 & 0 & 0 & -\frac{\partial N_{r}}{\partial y} \\
0 & 0 & 0 & -\frac{\partial N_{r}}{\partial y} & -\frac{\partial N_{r}}{\partial x} \\
0 & 0 & \frac{\partial N_{r}}{\partial x} & -N_{r} & 0 \\
0 & 0 & \frac{\partial N_{r}}{\partial y} & 0 & -N_{r}
\end{array}\right]}
\end{aligned}
$$




$$
\left[D_{p}\right]=\left[\begin{array}{cccccccc}
\frac{E t}{1-v^{2}} & \frac{v E t}{1-v^{2}} & 0 & 0 & 0 & 0 & 0 & 0 \\
\frac{v E t}{1-v^{2}} & \frac{E t}{1-v^{2}} & 0 & 0 & 0 & 0 & 0 & 0 \\
0 & 0 & \frac{E t}{2(1+v)} & 0 & 0 & 0 & 0 & 0 \\
0 & 0 & 0 & \frac{E t^{3}}{12\left(1-v^{2}\right)} & \frac{v E t^{3}}{12\left(1-v^{2}\right)} & 0 & 0 & 0 \\
0 & 0 & 0 & \frac{v E t^{3}}{12\left(1-v^{2}\right)} & \frac{E t^{3}}{12\left(1-v^{2}\right)} & 0 & 0 & 0 \\
0 & 0 & 0 & 0 & 0 & \frac{E t^{3}}{24(1+v)} & 0 & 0 \\
0 & 0 & 0 & 0 & 0 & 0 & \frac{E t}{2.4(1+v)} & 0 \\
0 & 0 & 0 & 0 & 0 & 0 & 0 & \frac{E t}{2.4(1+v)}
\end{array}\right]
$$

The element mass matrix can be expressed in isoparametric coordinates as

$$
\begin{aligned}
& {\left[M_{p}\right]=\int_{-1}^{+1} \int_{-1}^{+1}[N]^{T}\left[m_{p}\right][N]\left|J_{p}\right| d \xi d \eta} \\
& {\left[m_{p}\right]=\left[\begin{array}{ccccc}
\rho t & 0 & 0 & 0 & 0 \\
0 & \rho t & 0 & 0 & 0 \\
0 & 0 & \rho t & 0 & 0 \\
0 & 0 & 0 & \frac{\rho t^{3}}{12} & 0 \\
0 & 0 & 0 & 0 & \frac{\rho t^{3}}{12}
\end{array}\right]}
\end{aligned}
$$

When expressed in isoparametric coordinates the geometric stiffness matrix can be expressed as

$$
\left[K_{G p}\right]=\int_{-1}^{+1} \int_{-1}^{+1}\left[B_{G p}\right]^{T}\left[\sigma_{p}\right]\left[B_{G p}\right]\left|J_{p}\right| d \xi d \eta
$$

where $\quad\left[B_{G P}\right]=\left[\begin{array}{llllll}{\left[B_{G P}\right]_{1}} & {\left[B_{G P}\right]_{2}} & \cdots & {\left[B_{G P}\right]_{r}} & \cdots & {\left[B_{G P}\right]_{9}}\end{array}\right]$,

and

$$
\begin{aligned}
& \left\{\sigma_{p}\right\}=\left[D_{p}\right]\left\{\varepsilon_{p}\right\}, \\
& \left\{\sigma_{p}\right\}^{T}=\left\{\begin{array}{lllll}
\sigma_{x} & \sigma_{y} & \tau_{x y} & \tau_{z x} & \tau_{z y}
\end{array}\right\},
\end{aligned}
$$




$$
\left[B_{G_{P}}\right]_{r}=\left[\begin{array}{ccccc}
0 & 0 & \frac{\partial N_{r}}{\partial x} & 0 & 0 \\
0 & 0 & \frac{\partial N_{r}}{\partial y} & 0 & 0 \\
0 & 0 & 0 & \frac{\partial N_{r}}{\partial x} & 0 \\
0 & 0 & 0 & 0 & \frac{\partial N_{r}}{\partial y} \\
0 & 0 & 0 & 0 & \frac{\partial N_{r}}{\partial x} \\
0 & 0 & 0 & \frac{\partial N_{r}}{\partial y} & 0
\end{array}\right] .
$$

Similar to the plate element, the elastic stiffness matrix $\left[K_{S}\right]$, geometric stiffness matrix $\left[K_{G S}\right]$ and mass matrix $\left[M_{S}\right]$ of a stiffener element placed anywhere within a plate element and oriented in the direction of $x$ may be derived. Element stiffness matrix, element mass matrix, geometric matrix for stiffener can be expressed as

$$
\begin{aligned}
& {\left[K_{S}\right]=\int_{-1}^{+1}\left[B_{S}\right]^{T}\left[D_{S}\right]\left[B_{S}\right]\left|J_{S}\right| d \xi,} \\
& {\left[M_{S}\right]=\int_{-1}^{+1}[N]^{T}\left[m_{S}\right][N]|J| d \xi,} \\
& {\left[K_{G S}\right]=\int_{-1}^{+1}\left[B_{G S}\right]^{T}\left[\sigma_{S}\right]\left[B_{G S}\right]\left|J_{S}\right| d \xi}
\end{aligned}
$$

where $\quad\left[B_{S}\right]_{r}=\left[\begin{array}{ccccc}\frac{\partial N_{r}}{\partial x} & 0 & 0 & 0 & 0 \\ 0 & 0 & 0 & -\frac{\partial N_{r}}{\partial x} & 0 \\ 0 & 0 & 0 & 0 & \frac{\partial N_{r}}{\partial x} \\ 0 & 0 & \frac{\partial N_{r}}{\partial x} & -N_{r} & 0\end{array}\right]$,

$$
\left[\overline{D_{S}}\right]=\left[\begin{array}{cccc}
E A_{S} & E F_{S} & 0 & 0 \\
E F_{S} & E S_{S} & 0 & 0 \\
0 & 0 & G T_{S} & 0 \\
0 & 0 & 0 & G A_{S} / 1.2
\end{array}\right],
$$




$$
\left[m_{S}\right]=\rho\left[\begin{array}{ccccc}
A_{S} & 0 & 0 & 0 & 0 \\
0 & A_{S} & 0 & 0 & 0 \\
0 & 0 & A_{S} & 0 & 0 \\
0 & 0 & 0 & F_{S} & 0 \\
0 & 0 & 0 & 0 & P_{S}
\end{array}\right]
$$

where $A_{S}$ is the area, $F_{S}$ is the first moment of the area about the reference plane, $S_{S}$ is the second moment of the area about the reference plane, $T_{S}$ is the torsion constant and $P_{S}$ is the polar moment of the area of the stiffener cross-section.

\section{Problem description}

The basic configuration of the problem considered here is a rectangular stiffened plate $(a x b)$ with stiffeners having a rectangular cutout of size $(g \times d)$ at the center subjected to in-plane partial edge loading at the plate boundary as shown in Fig.1. The choice of the stiffened plate with cutout geometry as a basic configuration has been made so that depending on the value of the cutout size $\left(\begin{array}{ll}g & d\end{array}\right)$, partial edge load, extent of load width and position, various problems of stiffened plates with or without cutout subjected to uniform or partial edge loading can be considered as special cases. The stiffened plates with stiffener and stiffener cross-section are shown in Fig.2. The loading applied is compressive in nature. All the boundaries of the plates are simply supported unless otherwise stated. The partial edge loadings have different locations relative to the lower edge. All the boundaries of the plates are simply supported unless otherwise stated. The length (a) of the stiffened plate considered above is varied keeping its other parameters unchanged. Numerical results are presented for isotropic stiffened plate with different boundary conditions. For comparison, the boundary conditions are considered as reported in the respective studies. Here the displacement components $\left(w, \theta_{X}, \theta_{y}\right)$ are set to be zero over the whole domain of the plate for pre buckling analysis thereby reducing the problem size considerably. However, in the case of partially compressed plates, out of plane displacements do appear. These out of plane displacements vanish only when the full edge is subjected to uniform or linearly varying compression. The width of the extent of partial edge load at one end from the lower edge is assumed as ' $c$ ' throughout the analysis.

The presence of the cutout in the plate produces stress concentrations and high stress gradients in the neighbourhood of the cutout, which calls for an extra fineness of the mesh in this zone in the finite element discretization.

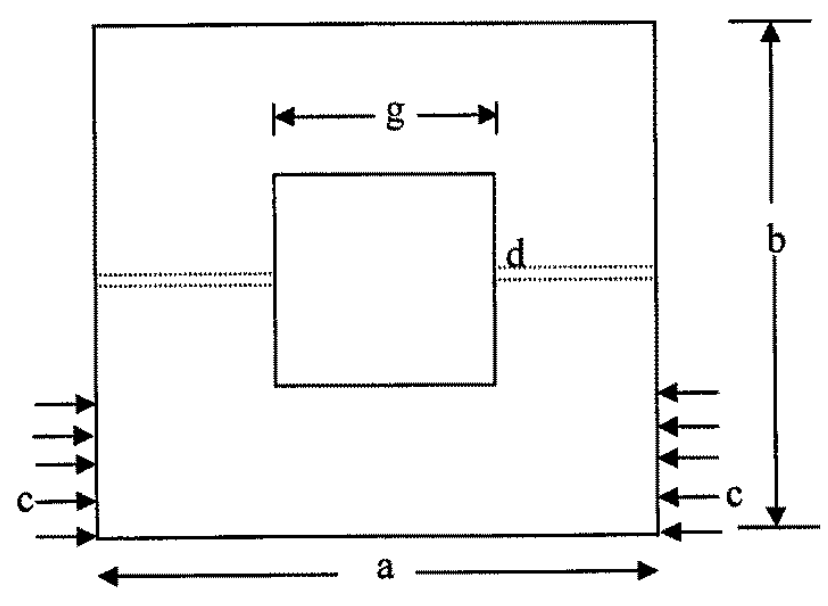

Fig.1. Stiffened plates with cutout under in plane partial edge loading at one end at plate boundary. 


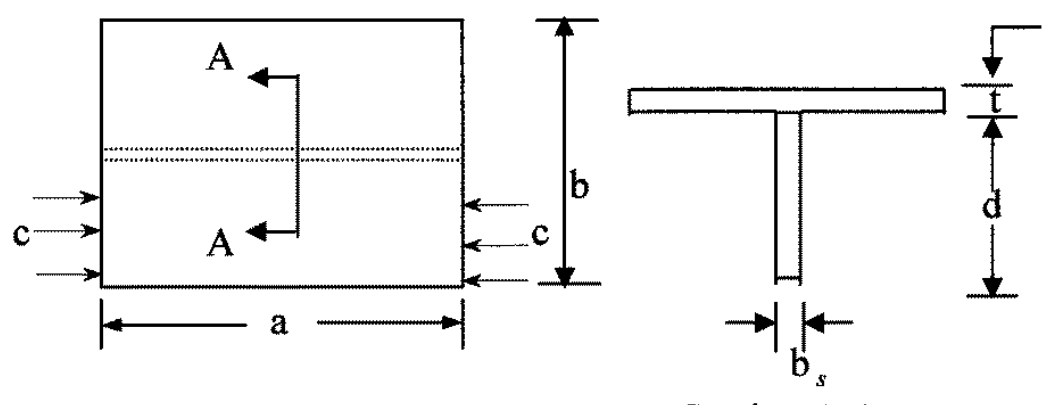

Section A A

Fig.2. Stiffened plate cross-section.

\section{Result and discussion}

The non-dimensionalisation of different parameters like vibration, buckling and excitation frequency for the dynamic stability analysis is taken as shown in Tab.1. The majority of the model parameters and results are presented in a non-dimensional form to make them independent of the plate size, thickness, material properties, etc for the convenience of the analysis.

Table1. Non-dimensionlisation of parameters.

\begin{tabular}{||c|c|c||}
\hline \hline & Term & Non-dimensionalisation values \\
\hline 1 & Frequencies of vibration $(\omega)$ & $\varpi b^{2} \sqrt{\rho t / D}$ \\
\hline \multirow{3}{*}{2} & $(1) \quad$ Duckling load $(\lambda)$ & $N_{X} b^{2} / \pi^{2} D$ \\
& $(2) \quad$ Concentrated load & $P_{c r} b / D$ \\
\hline 3 & Frequencies of excitation $(\Omega)$ & $\bar{\Omega} b^{2} \sqrt{\rho t / D}$ \\
\hline
\end{tabular}

where $D$ is the plate flexural rigidity, $D=E t^{3} / 12\left(1-v^{2}\right), P$ is the applied load, $P_{c r}$ is the buckling load, $\rho$ is the density of the plate material and $t$ is the plate thickness.

Assuming a general case of several longitudinal ribs and denoting by $E I_{S}$ the flexural rigidity of a stiffener at a distance $\left(D_{x}\right)$ from the edge $y=0$, the stiffener parameter terms are defined as: $\delta=A_{S} / b t=$ the ratio of the cross-sectional area of the stiffener to the plate, where $A_{S}$ is the area of the stiffener. $\gamma=E I_{S} / b D=$ Ratio of bending stiffness rigidity of stiffener to the plate, where $I_{S}$ is the moment of inertia of the stiffener cross-section about the reference axis. The frequency parameter is expressed in terms of a non-dimensional frequency parameter $(\omega)$ as: non-dimensional frequency, $\varpi=\omega b^{2} \sqrt{\rho h / D}$ which is a function of only the Poisson ratio and aspect ratios of the plates for a given cutouts configuration.

In addition, certain quantities are expressed as the ratio of that quantity to some reference quantity. In the discussion that follows, $S, C$ and $F$ denote simply supported, clamped and free edges respectively. The notation SCSF identifies a plate with the edges: $x=0, x=a, y=0, y=b$ having the boundary conditions in that order. Numerical results are presented for an isotropic unstiffened, stiffened plate with different boundary conditions. 
The problem of isotropic stiffened plates without cutout with uniform loading $(c / b=1.0)$ is investigated for buckling (static stability). Vibration characteristics for establishment of convergence and validity is discussed. Here the displacement components $\left(w, \theta_{X}, \theta_{y}\right)$ are set to be zero over the whole domain of the plate for pre buckling analysis thereby reducing the problem size considerably.

In a finite element analysis, it is desired to have the convergence studies to estimate the order of the mesh size necessary for the numerical solution. The plate area of interest is divided in $M x N$ finite element mesh, where $M$ is the number of divisions along the $x$-axis and $N$ is the number of divisions along the $y$-axis. The mesh division is generally uniform in both $x$ and $y$ directions. However to accommodate the mesh division at a desired edge location, the plate is divided into two zones and each zone is meshed separately with a uniform mesh. The actual values of $M$ and $N$ are based on the convergence studies. The mesh that has been used has been kept uniform throughout, not only from the point of view of ease of use, but also experience indicates that this tends to minimize numerical inaccuracy in computation. The in-plane movement at the boundaries of the plate has not been constrained.

\subsection{Convergence and validation}

For the validation of the buckling load parameter of stiffened plates subjected to partially distributed in-plane edge loading at one end at the plate boundary as shown in Fig.1, the analysis is carried out for $c / b=1$. Actually this corresponds to a fully loaded plate problem, for which analytical, finite element and other solutions are available in the literature. In the present case, the plate contains one stiffener as shown in Fig.2. By varying the stiffener parameters, the plate is analyzed taking $a / b=1.0$ (Fig.2) and the simply supported boundary condition at the four edges.

Results obtained in the present analysis are presented with the analytical solution of Timoshenko and Gere (1963) and finite element results of Mukherjee and Mukhopadhyay (1990) in Tab.2. It shows that the agreement between the results obtained from different sources is very good.

Table 2. Validation of buckling load parameter of a simply supported stiffened plates under distributed inplane edge loading. $(c / b=1)$.

\begin{tabular}{ccccccccc}
\hline & \multicolumn{3}{c}{$\gamma=5$} & \multicolumn{3}{c}{$\gamma=10$} \\
& \multicolumn{1}{c}{$\delta=0.05$} & \multicolumn{2}{c}{$\delta=0.1$} & \multicolumn{2}{c}{$\delta=0.05$} & \multicolumn{2}{c}{$\delta=0.10$} \\
\cline { 2 - 10 }$a / b$ & Present & A & Present & A & Present & B & Present & B \\
& & {$[1963]$} & & {$[1963]$} & & {$[1990]$} & & {$[1990]$} \\
\hline 1.0 & 11.98 & 12.0 & 11.03 & 11.11 & 15.99 & 16.00 & 15.99 & 16.0 \\
1.2 & 9.85 & 9.83 & 9.02 & 9.06 & 15.34 & 15.30 & 14.09 & 14.2 \\
1.6 & 8.17 & 8.01 & 7.35 & 7.38 & 11.43 & 11.40 & 10.48 & 10.5 \\
2.0 & 8.01 & 7.95 & 7.28 & 7.29 & 10.20 & 10.20 & 9.34 & 9.35 \\
3.0 & 8.31 & 8.31 & 7.67 & 7.62 & 12.03 & 12.0 & 11.10 & 11.1 \\
\hline
\end{tabular}

In table A is Timoshenko and Gere (1963) and B is Mukherjee and Mukhopadhyay (1990).

In order to validate the results of cutout, linear fundamental frequency parameters of a simply supported and clamped isotropic square plate with various sizes of rectangular cutout $(g / a)$ are computed and 
compared in Tab.3 with Mundkur et al. (1994) given in bracket, showing good agreement. Mundkur et al (1994) analyzed assuming boundary characteristics orthogonal polynomial functions, and assumed plate deflection shape functions satisfying the boundary conditions along the plate outer edges. The orthogonal polynomials are generated along both $x$ and $y$ directions.

Table 3. Comparison of natural frequency parameter.

\begin{tabular}{|c|c|c|c|c|}
\hline \multicolumn{5}{|c|}{ Natural frequency parameter $(\omega)$} \\
\hline & \multicolumn{2}{|c|}{ SSSS } & \multicolumn{2}{|c|}{$\mathrm{CCCC}$} \\
\hline$g / a$ & $\begin{array}{l}\text { Mundkur et al. } \\
\text { (1994) }\end{array}$ & Present & $\begin{array}{l}\text { Mundkur et al. } \\
\text { (1994) }\end{array}$ & Present \\
\hline 0.0 & 19.739 & 19.732 & 35.985 & 35.981 \\
\hline 0.167 & 20.070 & 19.87 & 37.425 & 36.06 \\
\hline 0.33 & 20.9633 & 20.12 & 43.867 & 43.02 \\
\hline 0.5 & 24.2434 & 24.24 & 65.715 & 65.27 \\
\hline
\end{tabular}

A free vibration analysis of a square plate with centrally stiffened opening analyzed by Paramasivam and Rao (1973) by the grid framework model and Shastry and Rao (1977) by the finite element model is carried out and presented in Tab.4 in the form of the fundamental frequency parameter $\omega$ for simply supported and clamped square plate.

Table 4. Convergence and validation of non-dimensional fundamental frequencies of a SSSS and CCCC unstiffened / stiffened square plates with cutout. $g / a=0.5, a / b=1, b / h=100, \gamma=10$.

\begin{tabular}{|c|c|c|c|c|c|c|c|}
\hline \multirow{3}{*}{$\begin{array}{l}\text { Boundary } \\
\text { condition }\end{array}$} & \multirow{3}{*}{$\begin{array}{c}\text { Types of } \\
\text { plates }\end{array}$} & \multicolumn{6}{|c|}{ Fundamental frequency parameter $(\omega)$} \\
\hline & & \multicolumn{4}{|c|}{ Mesh size } & \multirow{2}{*}{$\begin{array}{c}\text { Paramsivam } \\
(1977)\end{array}$} & \multirow{2}{*}{$\begin{array}{l}\text { Shastry } \\
(1973)\end{array}$} \\
\hline & & $6 \times 6$ & $8 \times 8$ & $10 \times 10$ & $12 \times 12$ & & \\
\hline \multirow{2}{*}{$\begin{array}{c}\text { Simply } \\
\text { supported }\end{array}$} & Unstiffened & 26.87 & 26.57 & 26.42 & 26.48 & 26.80 & 25.45 \\
\hline & Stiffened & 30.79 & 30.12 & 30.02 & 30.02 & 29.02 & 28.72 \\
\hline \multirow{3}{*}{ Clamped } & Unstiffened & 66.92 & 66.80 & 66.80 & 66.81 & 68.67 & 57.25 \\
\hline & & & & & & & \\
\hline & Stiffened & 71.09 & 70.98 & 70.57 & 70.56 & 70.87 & 68.62 \\
\hline
\end{tabular}

The parameter $\mathrm{E}_{\mathrm{S}} I_{S} / D L$ is taken as 10 , where, $\mathrm{E}_{\mathrm{S}}$ is Young's modulus of the stiffener material, $I_{S}$ is the moment of inertia of the stiffener and $D$ is the plate flexural rigidity.

The present results agree well with the result obtained by Shastry and Rao (1977) and by Paramasivam and Rao (1973). Torsional rigidity of the stiffeners and the effect of lateral deformation have been neglected. The comparison of these results with those in the literature shows the reliability and accuracy of the present formulation and program. It is observed that the frequency parameter results converged and agreed well with the above reference. As the convergence study shows a mesh size of $10 \times 10$ is sufficient enough to get a reasonable order of accuracy. The analysis in the subsequent problems is carried out with this mesh size. The predicted natural frequencies for square plates with cutouts are also compared with the reported value in the literature for the CSCS square plate. The first four natural frequency parameters are obtained for different sizes of cutouts in Tab.5. The corresponding boundary characteristics orthogonal 
polynomial functional results for the CSCS square plate are shown within parentheses. The predicted changes in the frequency agree with the finite element results of Mundkur (1994) showing an initial decreasing trend with increased cutout sizes.

Table 5. Linear fundamental frequencies of a CSCS square plate with square cutout.

\begin{tabular}{ccccccc}
\hline \multicolumn{7}{c}{ Natural frequency parameter $(\omega)$} \\
\hline Mode & \multicolumn{7}{c}{ Cut out ratio $(g / a)$} \\
\cline { 2 - 7 } & 0 & 0.1 & 0.2 & 0.3 & 0.5 & 0.8 \\
\hline \multirow{2}{*}{1} & 29.02 & 29.32 & 30.296 & 32.215 & 44.368 & 90.272 \\
& $(28.9509)$ & $(29.2675)$ & 30.2057 & $(32.1893)$ & $(44.3386)$ & $(90.2553)$ \\
2 & 54.86 & 54.87 & 54.9154 & 55.14 & 55.58 & 92.623 \\
& $(54.7432)$ & $(54.7512)$ & $(54.8577)$ & $(55.0372)$ & $(55.5415)$ & $(92.6066)$ \\
3 & 69.61 & 69.68 & 69.723 & 69.782 & 74.789 & 182.109 \\
& $(69.3270)$ & $(69.3416)$ & $(69.5036)$ & $(69.7469)$ & $(74.7403)$ & $(182.03)$ \\
4 & 94.765 & 94.714 & 92.412 & 91.397 & 94.312 & 188.612 \\
& $(94.5823)$ & $(93.9170)$ & $(92.3961)$ & $(91.3022)$ & $(94.2797)$ & $(188.5265)$ \\
& & & & &
\end{tabular}

The result of parentheses is from Mundkur (1994).

\subsection{Numerical results}

The vibration frequencies and buckling load parameter for various modes of rectangular stiffened plates with one central stiffener subjected to in-plane uniform uni-axial loading and partial edge loading at the plate boundary have been determined for simply supported edge conditions. The influence of the size of cutout and extent of the width of partial edge loading on the buckling and vibration behaviour of stiffened plates has been examined. Numerical results are presented for a range of hole to plate width ratios of 0 to 0.8 . The necessary data used are as follows: $a=600 \mathrm{~mm}, b=600 \mathrm{~mm}, t=1 \mathrm{~mm}, b_{S}=3.31 \mathrm{~mm}, d_{S}=20.2 \mathrm{~mm}$, $v=0.34, E=6.87 \times 10^{4} \mathrm{~N} / \mathrm{mm}^{2}, \rho=2.78 \times 10^{-6} \mathrm{Kg} / \mathrm{mm}^{3}$.

Fundamental frequencies are computed for a simply supported plate having one central stiffener with a central square cutout of different sizes $(g / a)$ subjected to uni-axial compressive force and the results are presented in Fig.3. Numerical results for the buckling load parameter for a stiffened square plate having one central stiffener with a central square cutout of different sizes subjected to uni-axial compressive force for boundary conditions (SSSS, SSCC) are shown in Fig.4.

It is observed from Fig. 3 that the frequency parameter increases with the increase of the cutout size. A similar behaviour was observed for higher modes of oscillation. The effects of cutout sizes on natural frequency for stiffened plates subjected to bi-axial force have also been studied and observed to be same as the uni-axial compressive force. It is observed from Fig.4 that the buckling load of the stiffened plate reduces with the increase in the size of the hole.

The influence of various parameters on buckling, vibration and dynamic stability characteristics of stiffened plates with cutout subjected to partial edge load at one end are studied. 


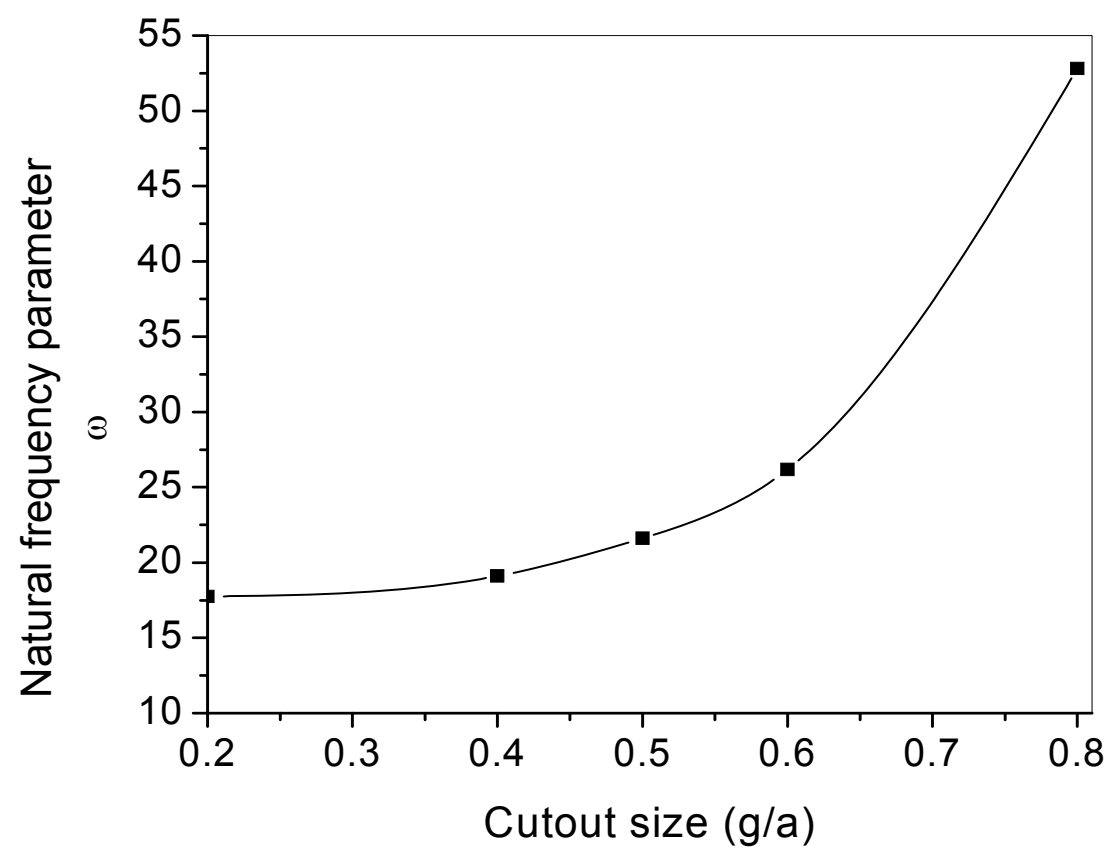

Fig.3. Frequency parameters for a simply supported stiffened square plate having one central stiffener with square cutout subjected to uni-axial compressive forces.

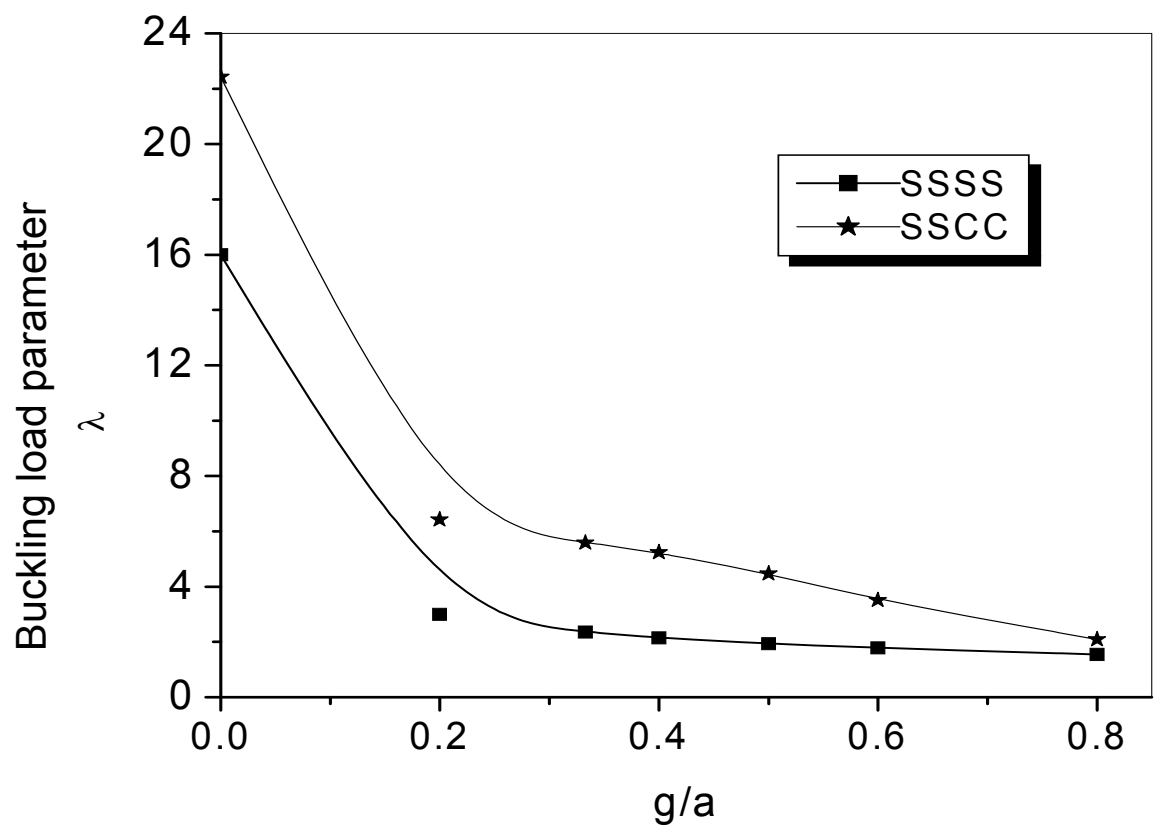

Fig.4. Buckling load parameter $(\lambda)$ vs hole/plate ratio $(g / a)$ for uni-axially loaded stiffened plate with one central stiffener $(\delta=0.1$ and $\gamma=10)$. 


\subsubsection{Buckling studies of stiffened plates with cutout}

Numerical results for the buckling load parameter are presented for a simply supported stiffened square plate having one central stiffener in three higher modes for the cases:

(i) Various central square cutout sizes $(g / a=0.2,0.4,0.6,0.8)$ and partial edge load width $(c / b=0.4)$ at one end in Fig.5 and (ii) For cutout of size $(g / a=0.4)$ at various load width $(c / b=0.2,0.4,0.5,0.6,0.8)$ in Fig.6.

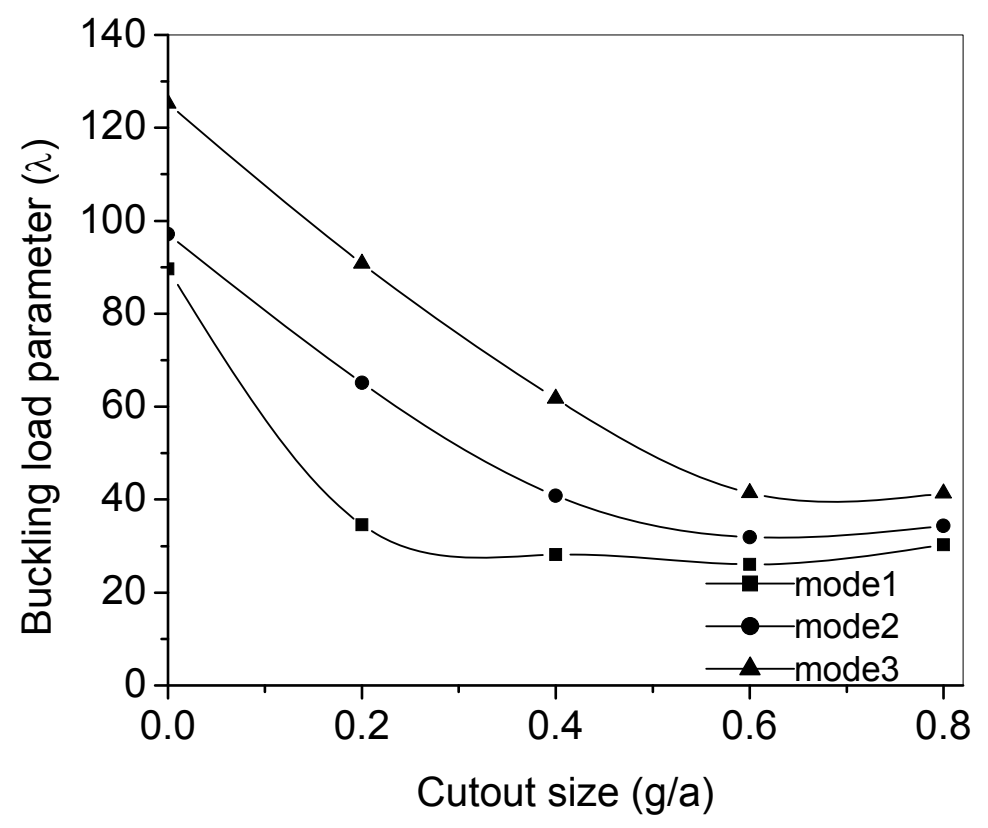

Fig.5. Effect of cutout $(g / a)$ on buckling load parameter for simply supported stiffened plate with cutout having one central stiffener subjected to partial edge load at one end. $a / b=1, c / b=0.4$.

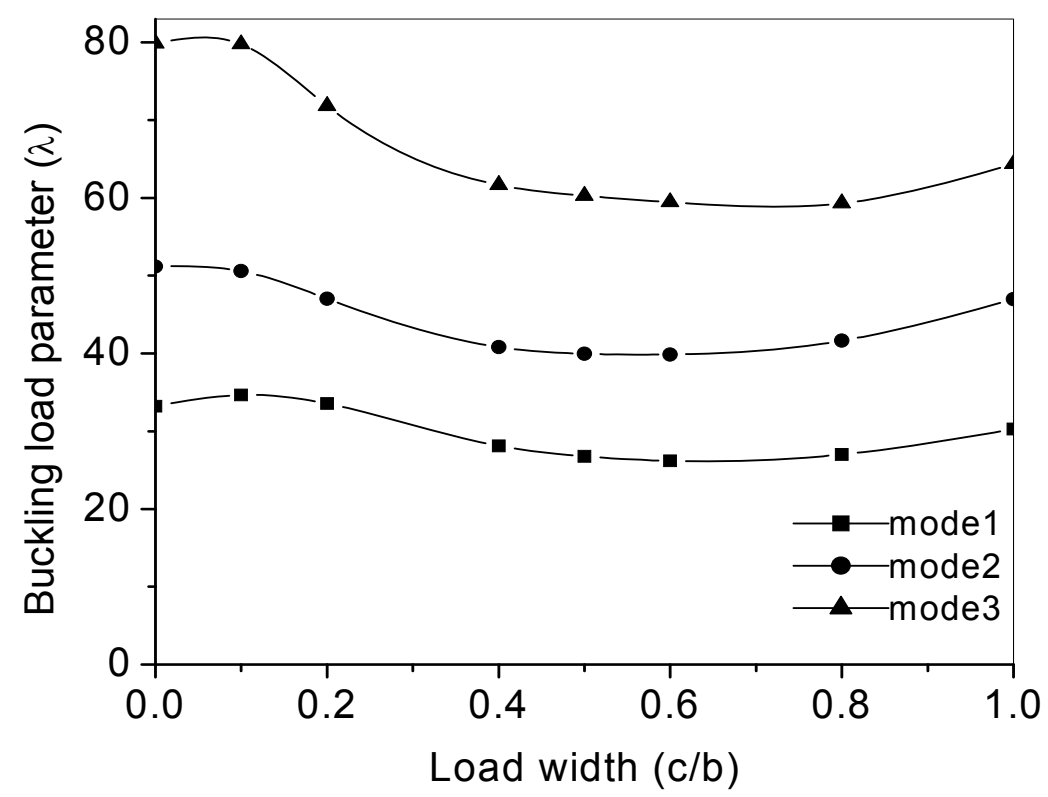

Fig.6. Effect of load width $(c / b)$ on buckling load parameter with load width $(c / b)$ for simply supported stiffened plate with cutout having one central stiffener subjected to partial edge load at one end. $g / a=0.4, a / b=1$. 
It is observed from Fig. 5 that buckling resistance decreases as the cutout size increases in all the modes. It is observed from Fig. 6 that buckling resistance decreases as the load bandwidth $(c / b)$ increases from 0 to 0.4 . This can be explained by the fact that as the load width increases, the edge restraint effect decreases and consequently the buckling load decreases. However, as the load width $(c / b)$ increases from 0.4 to the uniform full width $(c / b=1)$, no appreciable variation is noticed.

\subsubsection{Vibration studies of stiffened plates with cutout}

Numerical results for vibration frequencies are presented for a simply supported stiffened square plate having one central stiffener with a central square cutout. The variation of vibration frequencies with $P / P_{c r}$ is presented for the cases:

(i) for various cutout sizes $(g / a=0.2,0.4,0.6,0.8)$ and partial edge load width at one end $(c / b=0.4)$ in Fig.7 and (ii) for various load width $(c / b=0.2,0.4,0.6,0.8)$ with cutout size $(g / a=0.4)$ in Fig.8.

It is observed from Fig.7 that vibration frequencies increase with the increase of the cutout size $(g / a)$. It is observed from Fig. 8 that the effects of load width $(c / b)$ on vibration frequencies for the simply supported end condition are not very appreciable. It is also observed from Figs 7 and 8 that the frequency parameter decreases with the increase of $P / P_{c r}$ and it becomes zero at critical buckling.

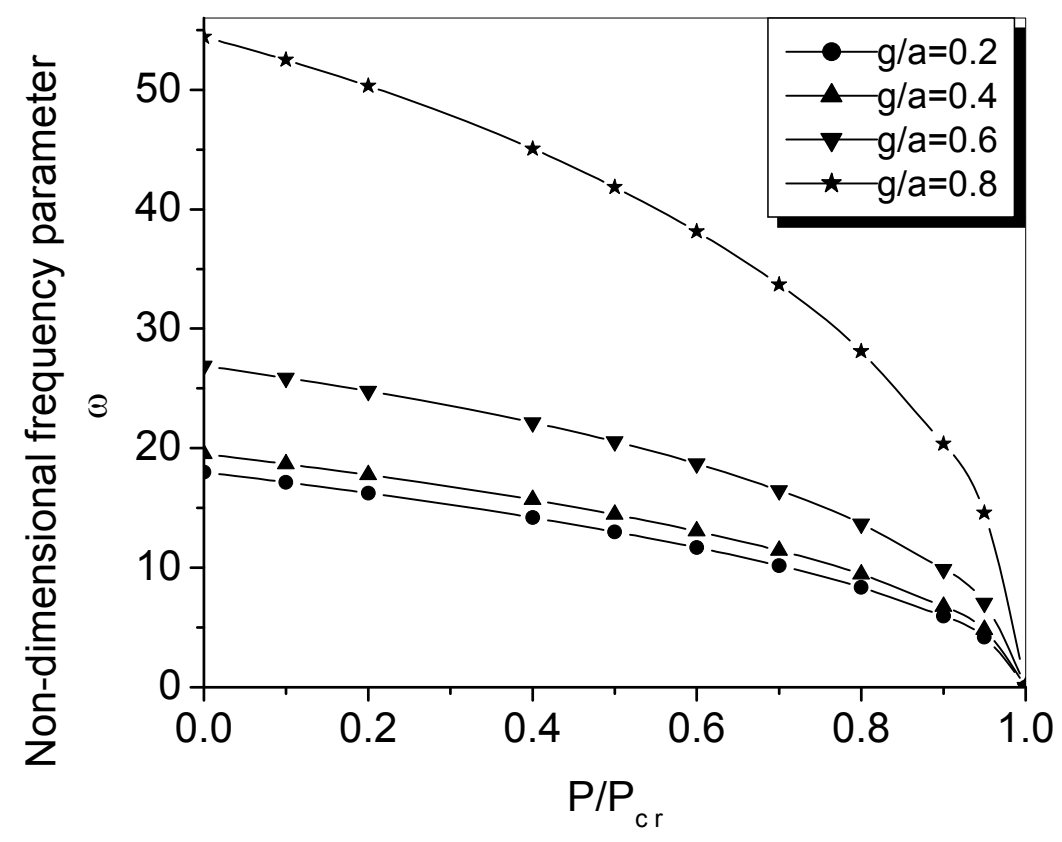

Fig.7. Variation of frequency parameter with $P / P_{c r}$ for various cutout sizes $(g / a)$ for simply supported stiffened square plate having one central stiffener subjected to partial edge load at one end. $c / b=0.4$. 


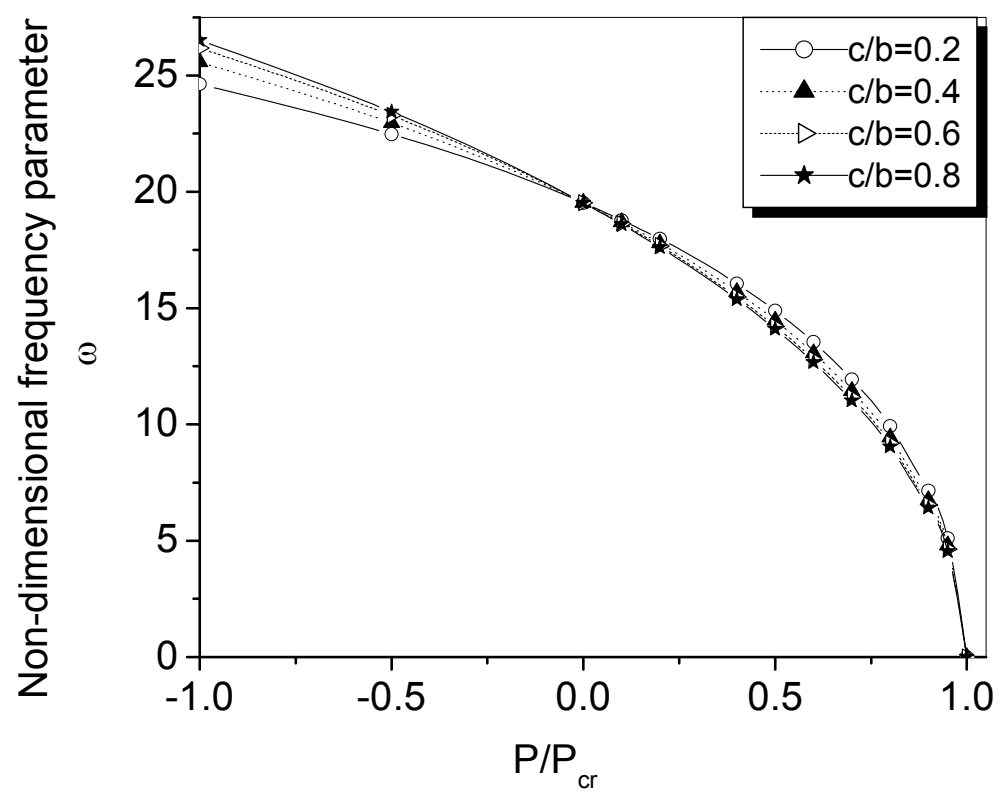

Fig.8. Variation of frequency parameter with $P / P_{c r}$ for various values of load width $(c / b)$ for simply supported stiffened square plate having one central stiffener subjected to partial edge load at one end. $g / a=0.4$.

\section{Conclusion}

Theoretical investigations of the buckling, vibration behaviour of stiffened plates with cutout, subjected to in-plane uniform and partial edge loadings at the plate boundary have been carried out using the finite element formulation and presented in this present work. Conclusions based on the results and discussions are summarized below.

The buckling and vibration behaviour for stiffened plates with cutout is more pronounced in comparison to the unstiffened plates. The cutouts have considerable influence on the buckling load parameter and vibration frequencies. Vibration frequencies increase with the increase of restraint at the edges. Vibration frequencies increase with the increase of the cutout size $(\mathrm{g} / \mathrm{a})$. A similar behaviour is also observed for higher modes of oscillation.

The vibration frequency parameter decreases with the increase of the load factor. The frequency parameter values increase with the increase of the cutout size. The rate of increase of frequencies also increases with the increase in the size of the hole.

The buckling load parameter of a stiffened plate with cutout reduces with an increase in the size of the cutouts. The stiffened plates are less susceptible to buckling under the localized loading near the ends of the loaded edges. As the load width increases, the edge restraint effect decreases and consequently the buckling load decreases. The effects of load width $(c / b)$ on vibration frequencies for the simply supported end condition are not very appreciable. The frequency parameter decreases with the increase of $P / P_{c r}$ and it becomes zero at critical buckling

Buckling and vibration behaviour of the stiffened plates with/ without cutout is greatly influenced by the stiffener parameters, the type and position of loads. So the designer has to be cautious while dealing with structures subjected to non-uniform loading. This can be used to the advantage of tailoring during design of stiffened plate structures. 


\section{Nomenclature}

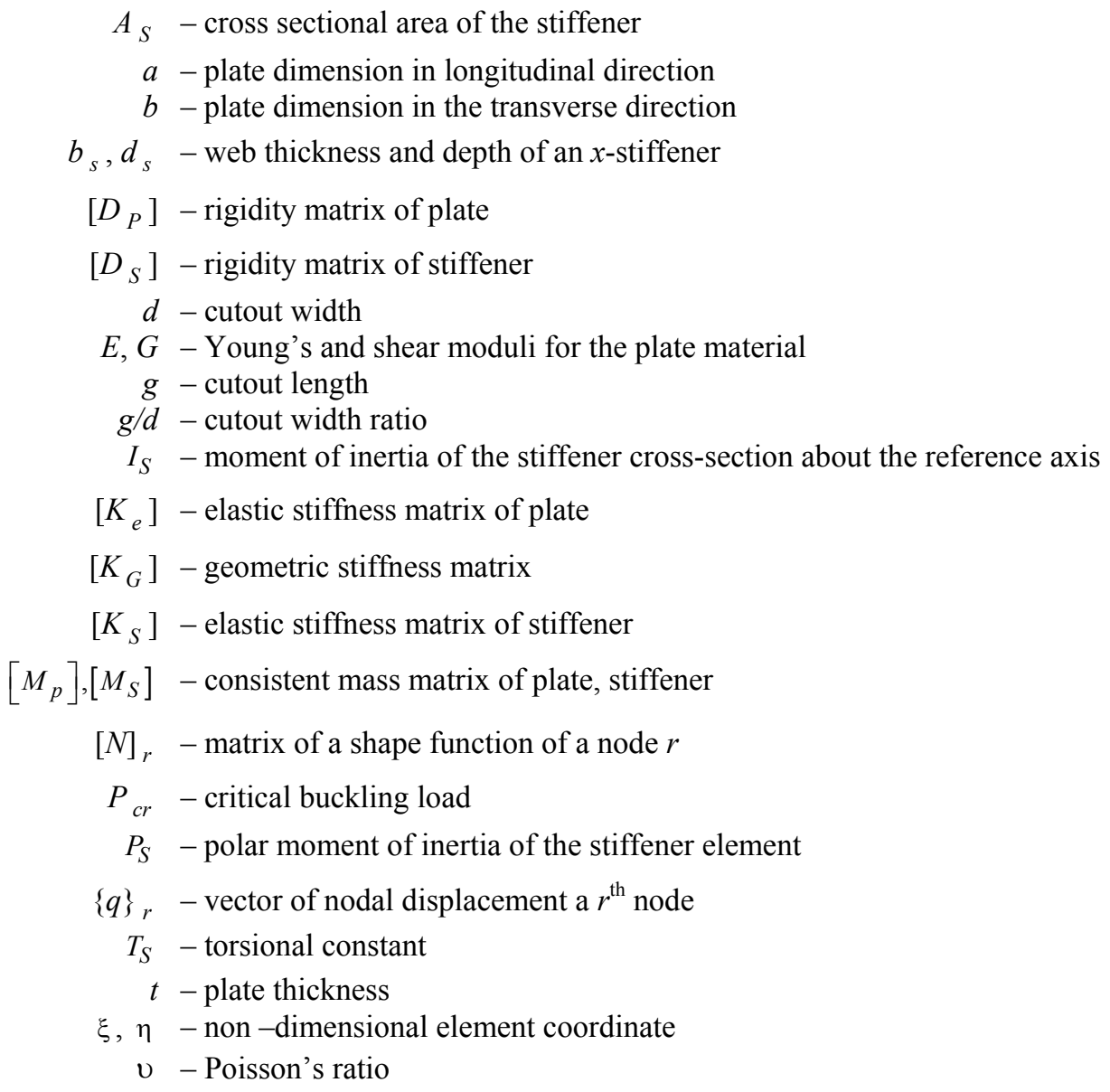

\section{References}

Ali R. and Atwal S.J. (1980): Prediction of natural frequencies of rectangular plates with rectangular cutouts. Computer and Structures, vol.12, pp.819-823.

Corr R.B. and Jennings A.A. (1976): Simultaneous iteration for symmetric eigen value problem. - Int. Journal of Numerical Method Engng., vol.10, pp.647-663.

Dickinson S.M. and Kalidas M.M. (1981): Vibration and buckling calculation for rectangular plates subjected to complicated in-plane stress distribution by using numerical integration in a Rayleigh- Ritz analysis. - Journal of Sound and Vibration, vol.75, pp.151-162.

Deolasi P.K., Datta P.K. and Prabhakar D.L. (1995): Buckling and vibration of rectangular plates subjected to partial edge loading (Compression or tension). - Journal of Structural Engineering, vol.22, No.3, pp.135-144.

Lam K.Y. and Hung K.C (1990): Vibration study on plates with stiffened openings using orthogonal polynomials and partitioning method. - Computer and Structures, vol.33, No.3, pp.295-301.

Leggett D.M.A. (1937): The effect of two isolated forces on the elastic stability of a flat rectangular plate. -Proceeding of the Cambridge Philosophical Society, vol.33, pp.325-329.

Mei C. and Yang T.Y. (1972): Free vibration of finite element plates subjected to complex middle plane force system.Journal of Sound and Vibration, vol.23, No.2, pp.145-156. 
Monahan I.J., Nemergut P.J. and Maddux G.E. (1970): Natural frequencies and mode shapes of plates with interior cutouts. - The Shock and Vibration Bulletin, vol.41, pp.37-49.

Mukhopadhyay M. and Mukharjee (1990): A finite element buckling analysis of stiffened plates. - Computer and Structures, vol.34, No.6, pp.795-803.

Mundkur G., Bhat R.B. and Neria S. (1994): Vibration of plates with cutouts using boundary characteristics orthogonal polynomial functions in the Rayleigh-Ritz method. - Journal of Sound and Vibration, vol.176, No.1, pp.136-144.

Paramsivam P. and Sridhar Rao J.K. (1969): Free vibration of rectangular plates of abruptly varying stiffnesses. International Journal of Mechanical Sciences, vol.11, pp.885-895.

Paramasivam P. (1973): Free vibration of square plate with square openings. - Journal of Sound and Vibration, vol.30, No.2, pp.173-178.

Ritchie D. and Rhodes J. (1975): Buckling and post-buckling behaviour of plates with holes. - Aeronautical Quarterly, vol.24, pp.281-296.

Shastry B.P. and Rao G.V. (1977): Vibration of thin rectangular plates with arbitrary oriented stiffeners. - Computer and Structures, vol.7, pp.627-629.

Sivasubramonian B., Kulkarni A.M. and Rao G.V. (1997): A free vibration of curved panels with cutout. - Journal of Sound and Vibration, vol.20, No.2, pp.227-234.

Srivastava A.K.L., Datta P.K. and Sheikh A.H. (2003): Vibration and dynamic instability of stiffened plates subjected to in-plane harmonic edge loading. - International Journal of Structural Stability and Dynamics, vol.2, No.2, pp.185206.

Sundersasan P., Singh G. and Rao G.V. (1998): Buckling of moderately thick rectangular composite plates subjected to partial edge compression. - International Journal of Mechanical Sciences, vol.40, No.11, pp.1105-1117.

Timoshenko S.P. and Gere J.M. (1963): Theory of Elastic stability. (Second edition). - New York: McGraw-Hill.

Uenoya M. and Redwood R.G. (1986): Elastic-plastic shear buckling of square plates with circular holes. - Computer and Structures, vol.22, No.4, pp.589-594.

Yamaki N. (1953): Buckling of rectangular plate under locally distributed forces applied on the two opposite edges. Report of the institute of High Speed Mechanics, Tohoko University, Japan, 26: 71-87 and No 27: 89-98.

Received: May 4, 2012

Revised: January 12, 2013 